\section{Für eine Regionalisierung des Gesundheitswesens in der Schweiz}

\author{
T. Fröhlich
}

Unser Gesundheitswesen ist eines der besten, wenn nicht sogar das beste in der Welt, aber offenbar doch zu teuer. So hat sich die Kostenreduktion sukzessive zum Primärziel aller Anstrengungen im Gesundheitswesen entwickelt.

Aufgrund eigener Erfahrungen während 31 bzw. 20 Jahre als niedergelassener Internist (Grundversorger) und Kantonsarzt Schaffhausen im Nebenamt versuche ich, in Ergänzung zu J. H. Dunant [1], Möglichkeiten für Verbesserungen aufzuzeigen. Im Interesse der Lesbarkeit wird im Zweifel die männliche Form verwendet.

Als Folge der kantonalen Hoheit im Gesundheitswesen wird das obligatorisch versicherte Bevölkerungskollektiv der Schweiz in 26 sehr unterschiedlich grosse Unterkollektive aufgeteilt. Diese kantonalen Unterkollektive sind aber vielfach zu klein, um statistisch relevant abgestützte Berechnungen der Prämien zu erlauben. Auch wird so die im KVG angestrebte Solidarität, vor allem in mittleren und kleinen Kantonen, weitgehend illusorisch.

Zudem sind sie für spitzenmedizinische Leistungen an ihrer Bevölkerung auf den guten Willen ihrer grösseren und besser ausgerüsteten Nachbarn angewiesen und müssen aufgrund des KVG zur Abklärung der Indikation jeder einzelnen ausserkantonalen Hospitalisation eine aufwendige Bürokratie unterhalten.

Bevor nicht sämtliche Möglichkeiten der Rationalisierung des Gesundheitswesens ausgeschöpft sind, darf im Interesse aller Bevölkerungsgruppen von Rationierung medizinischer Leistungen nicht die Rede sein, auch wenn die Rationalisierung den Abschied von vielen vertrauten, aber zunehmend teuren Strukturen erfordert. Aber gerade die Kantone könnten die Rationalisierung vorantreiben. Rationierungen ihrerseits müssten ja zuerst vom eidgenössischen Parlament beschlossen und letztlich vom Souverän akzeptiert werden.

Korrespondenz:

Dr. med. T. Fröhlich

Dorfstrasse 25

CH-8234 Stetten
Ohne neue politische Aussage bleiben aber die bisherigen Aufträge und Pflichten im Gesundheitswesen, insbesondere jene der Ärzte und Spitäler, gemäss jetzt gültigem KVG unverändert - und die Kosten steigen weiter. Also müssen die Politiker endlich grundsätzliche Überlegungen anstellen und nicht in Einzelfällen pragmatisch - dafür aber medienwirksam - entscheiden

Die grundlegenden Vorteile unseres föderalistischen Staates müssen deswegen keinesfalls aufgegeben werden, wir müssten nur seine Möglichkeiten besser nutzen. Diesem Ziel dienen folgende Vorschläge.

\section{Regionalisierung des Gesundheitswesens}

Das Gesundheitswesen wird mittels interkantonaler Verträge regionalisiert, die Kantons- bzw. Gemeindegrenzen entfallen für diesen Bereich, ohne dass die politischen Grundstrukturen geändert werden.

Denkbar sind in der Schweiz 5-6 Regionen mit koordiniertem Spital- bzw. Gesundheitswesen, wobei die Grenzen der Regionen nicht unbedingt mit den Kantonsgrenzen zusammenfallen müssen. An den interregionalen Grenzen allenfalls auftretende Probleme werden lokal und gemäss den Wünschen der betroffenen Bevölkerung gelöst. Das so geschaffene regionale Kollektiv der Grundversicherten wird nun gross genug, um in einem grösseren "Markt» echte Solidarität sicherzustellen und Kosten für alle zu verringern.

Jede Region enthält:

- wenigstens 1 Spitzenspital

(Universitätsklinik oder ähnliches Zentrum);

- 1 bis mehrere Kantonsspitäler;

- 1 bis mehrere Regional- bzw. Bezirksspitäler (heutige Nomenklatur).

Innerhalb der Regionen sind Arzt- und Spitalwahl frei (Wettbewerb auch der Spitäler).

Zusätzlich kommen im ambulanten wie im stationären Gesundheitswesen je ein einheitlicher Tarif (TARMED) und Taxpunktwert zur Anwendung. Die Aufgaben der Krankenkassen bleiben dabei unverändert. Aber als Folge stehen für die Berechnung der Prämien statistisch repräsentative Zahlen aus vergleichbaren Arztpraxen, Ambulatorien und Spitälern als Grundlage zur Verfügung.

Die Spitäler einer Region werden durch die beteiligten Kantone und Krankenkassen entsprechend ihren heutigen Aufwendungen für das Gesundheitswesen gemeinsam finanziert: Gesundheitskostenpool. Jedes Spital erhält einen stufengerechten, klar umschriebenen Leistungsauftrag und ein entsprechendes Globalbudget. Dafür sind dann zu schaffende regionale Führungsstrukturen verantwortlich. Diese führen auch Verhandlungen mit andern Partnern im Gesundheitswesen (Krankenkassen und anderen). 


\section{Ambulatorien der Spitäler}

Einzelne Kantone, und zwar vor allem die kleineren, erliegen zur Vermeidung ausserkantonaler Hospitalisationen heute gelegentlich der Versuchung, unter Erweiterung ihres ursprünglichen Auftrages zusätzliche Einrichtungen und Apparaturen zu beschaffen und so die diagnostischen und therapeutischen Möglichkeiten der eigenen Spitäler zu verbessern. Um diese dann wegen der zwangsläufig kleinen Fallzahlen besser auszulasten, werden die primär stationären Angebote sekundär auch auf den spitalexternen Bereich ausgedehnt. Dadurch konkurrenzieren die Spitäler durch ihre Ambulatorien die verschiedenen Hausarztsysteme, ohne aber das gesamte Angebot der Hausärzte an ambulanten Dienstleistungen abzudecken. Damit unterlaufen sie die Sparanstrengungen der Hausarztsysteme.

Ferner trennen die Spitäler weder in Budget noch Rechnung den stationären Bereich streng von Aufwand und Ertrag der Ambulatorien, wodurch die öffentliche Hand auch Leistungen mitfinanziert, die eigentlich nicht spitalgebunden sind.

Daher werden zur Verbesserung der Situation innerhalb der Regionen die Ambulatorien der Spitäler als Facharztpraxen eingestuft, deren Leistungen eine Zuweisung durch einen Grundversorger voraussetzen (Notfälle ausgenommen).

Sie rechnen ihre Leistungen zu den gleichen Bedingungen ab wie die entsprechenden niedergelassenen Fachärzte und weisen sie getrennt vom stationären Bereich aus. So entstehen zum Vorteil der Versicherten faire Wettbewerbsbedingungen zwischen Spitalambulatorien und niedergelassener Ärzteschaft.

\section{Abrechnungsmodus}

Der heute von Kanton zu Kanton unterschiedliche Abrechnungsmodus der Krankenkassen mit den Leistungserbringern ("tiers payant" bzw. "tiers garant») erschwert jeden interkantonalen Vergleich. Das Problem wird noch kompliziert durch die Tatsache, dass heute auch innerhalb des gleichen Kantons die niedergelassenen Leistungserbringer aller Art sowie die Spitalambulatorien nicht alle den gleichen Zahlmodus haben. Die Verwirrung ist komplett und treibt erst noch die Kosten in die Höhe.

Zur Verbesserung wird der Zahlmodus der Krankenkassen daher im Gesundheitswesen der Region gegenüber allen Leistungserbringern vereinheitlicht: "tiers garant" oder "tiers payant». Das KVG sieht auch beide Varianten vor. Im Interesse des Kostenbewusstseins bei Patienten und Leistungserbringern einerseits und der Unabhängigkeit der Leistungserbringer von den Kostenträgern andrerseits ist das System des "tiers garant" vorzuziehen.

Der "tiers garant" wird daher in den Regionen verbindlich festgelegt. Die dann gewinnbaren Daten aller Beteiligten werden - endlich - echte Vergleiche von Region zu Region erlauben. Jede administrative Vereinfachung spart Prämienanteile.
Daher gilt der "tiers garant" auch in der Versorgung mit Medikamenten: Der Patient erhält die Kosten minus Selbstbehalt usw. nur nach Vorweisen bzw. Einschicken der Quittung des Apothekers zurück. Dies wird das Kostenbewusstsein des Grundversicherten erhöhen, ihn zur Therapietreue anhalten und gleichzeitig die Medikamentenkosten vermindern. Noch sparsamer wäre ja bekanntlich die DMA, aber an den heute gültigen Regelungen der DMA wird vorläufig nichts geändert.

\section{Kontrolle}

Für die Überprüfung der Wirtschaftlichkeit innerhalb der Region werden aus Vertretern aller Partner gemischte Gremien mit neutralem Vorsitz berufen. Die Kontrolle der medizinischen Qualität (fachlich und menschlich) muss Sache der Fachverbände bleiben.

\section{Krankenkassen}

Entscheide von Vertrauensärzten der Krankenkassen können zuerst an eine nicht gerichtliche Fachinstanz zum Rekurs weitergezogen werden (Region). Zudem vergüten die Krankenkassen ihren Versicherten bezogene Leistungen ausschliesslich aufgrund nachweislich bezahlter Rechnungen ("tiers garant»).

\section{Schularztdienste}

Die bisherigen Schularztdienste der Kantone werden im Leistungsumfang wenigstens regional vereinheitlicht und auf nachgewiesenermassen nutzbringende Massnahmen reduziert (Regionale SDK/EDK). Nur solche sind präventivmedizinisch gerechtfertigt und langfristig kostenwirksam.

\section{Austausch von Informationen unter den Ärzten}

Die gegenseitige ärztliche Information bei ambulanter Zuweisung oder Einweisung ins Spital bzw. Rücküberweisung an den Hausarzt muss, im ausdrücklichen Einverständnis des Patienten, offen, zeitgerecht und vollumfänglich erfolgen. Dies gehört zum Dienst am Patienten, dem sich alle Ärzte, wo immer sie auch tätig sind, verpflichtet haben. Die Informationspolitik im Gesundheitswesen ist ein Führungsproblem und verdient auch die Aufmerksamkeit der ärztlichen Standes- und Spitalorganisationen.

Damit dieser Informationsfluss aber den angestrebten Nutzen bringt, sind die Akzeptanz der vom anderen Arzt sowohl klinisch wie technisch erhobenen Befunde sowie der Verzicht auf medizinisch nicht neu indizierte Wiederholungen von technischen Untersuchungen Bedingung sine qua non.

Die geforderte Akzeptanz "fremder" Resultate setzt aber ihrerseits auch gegenseitige Transparenz voraus, das heisst hüben und drüben Kenntnis über 
die technischen Einrichtungen und die verwendeten Labormethoden. Diese Art der Information in gegenseitiger Akzeptanz und Transparenz spart Kosten und verringert dem Patienten Selbstbehalt und Prämien.

\section{Zusammenarbeit mit Partnern im Gesundheitswesen}

Auch die gute Zusammenarbeit mit anderen qualifizierten Leistungserbringern im Gesundheitswesen, wie Physiotherapeuten, Chiropraktikern usw., insbesondere aber mit den Apothekern, liegt im Interesse des Patienten, der sich uns anvertraut. Eine korrekte Verordnung (Rezept) und die entsprechende Abgabe des Medikamentes mit sofortiger gegenseitiger Kontaktnahme im Zweifelsfall erleichtern diesen für den Patienten wesentlichen Informationstransfer.
Der korrekte Umgang mit den einzelnen Versicherern ist auch Dienst am Patienten und implizite in seinem dem Arzt erteilten "Auftrag" enthalten. Allerdings muss der Tendenz der Versicherer zur Durchlöcherung des Patientengeheimnisses in all ihren Formen entgegengetreten werden. Salus aegroti suprema lex darf nicht nur in Praxen und Spitälern gelten, sondern muss auch für die Versicherer Verpflichtung sein.

\section{Literatur}

1 Dunant JH. Gesundheitswesen: Qualität und Ökonomie sind vereinbar. Schweiz Ärztezeitung 2001;82(28):1516-8. 